\title{
Conversion $\mathrm{X}$ dans l'interaction laser matière
}

\author{
J.-L. Bocher
}

CEA/Centre d'Etudes de Limeil-Valenton, 94195 Villeneuve Saint Georges Cedex, France

\begin{abstract}
The interaction of a high power laser beam with matter results in a highly ionized medium, called a plasma. For target irradiations higher than $10^{13} \mathrm{Wcm}^{-2}$, the energy density supplied to the target is such that the plasma temperature exceeds one million degrees. The major part of energy is then thermal but also radiative and the maximum of the emission $\left(1 \AA<\lambda_{\max }<100 \AA\right)$ appears in the $X$-ray range. It is important to study this radiation, because it is the image of the physical processes which control the interaction between electromagnetic wave and matter and it contributes for an important part to the energetic balance. Furthermore, this $X$-ray emission can be used as a source in the studies of inertial confinement by indirect drive, in atomic physics, in absorption spectroscopy, in X-ray shadowgraphy, in X-ray laser, and industrial applications like lithography. The studies of the conversion efficiency of laser energy in $X$-ray energy have shown the advantage of short laser wawelengths, of high $\mathrm{Z}$ materials, and the appearance of a maximum of this conversion as a function of laser irradiance. For gold targets, $0.35 \mu \mathrm{m}$ laser wavelength and $10^{14} \mathrm{Wcm}^{-2}$ laser flux, the $X$-ray conversion efficiency can reach $60 \%$.
\end{abstract}

L'interaction d'un faisceau laser de grande puissance avec la matiere s'accompagne de l'ionisation de celle-ci et conduit à la formation d'un plasma[1].

Pour des éclairements de cibles supérieurs à $10^{13} \mathrm{~W} \mathrm{~cm}^{-2}$ la densité d'énergie injectée dans la cible est telle que la température du plasma dépasse le million de degrés. De ce fait la majeure partie de l'énergie se retrouve sous forme thermique et radiative, le maximum de l'émission $\left(1 \AA<\lambda_{\max }<100 \AA\right)$ se situant dans le domaine du rayonnement $X$. Il est nécessaire d'étudier ce rayonnement car il est le reflet des processus physiques qui gouvernent l'interaction de l'onde électromagnétique avec la matière et il contribue pour une part importante au bilan énergétique. De plus ce rayonnement $X$ peut être utilisé dans les études de fusion thermonucléaire, pour la spectroscopie d'absorption, pour la radiographie $X$, ou d'autres applications plus industrielles telles que la lithographie. 
L'étude des variations du taux de conversion ( rapport de l'énergie $X$ émise à l'énergie laser incidente $E_{X} / E_{1}$ ) du rayonnement laser en rayonnement $X$ montre l'intérêt, pour porter ce taux à son maximum, des faibles longueurs d'onde laser et des matériaux à numéro atomique (Z) élevés (fig1). En outre il existe un maximum de conversion en fonction de l'éclairement laser; pour une cible d'or, une longueur d'onde du laser de $0,35 \mu \mathrm{m}$ et un éclairement de $10^{14} \mathrm{Wcm}^{-2}$ il peut atteindre $60 \%$ (fig2). L'utilisation de matériaux de numéros atomiques différents permet d'adapter la forme du spectre à l'utilisation envisagée.

Le principal résultat obtenu avec les feuilles minces a été la mise en évidence expérimentale d'un maximum du taux de conversion $X$ face arrière, qui donc n'a pas été éclairée par le laser, en fonction de l'épaisseur de la cible, résultat qui avait été prévu théoriquement. On montre qu'il y a une thermalisation $d u$ rayonnement, c'est à dire d'une disparition des émissions de raies, la forme du spectre se rapproche alors de celui d'un corps noir.

L'ensemble des résultats obtenus depuis plusieurs années montre une bonne cohérence entre les différents laboratoires et permet d'avoir une vue plus précise

des émissions attendues pour l'ensemble des paramètres de l'interaction. Ils ont permis une meilleure connaissance des problèmes de l'hydrodynamique radiative dans les plasmas créés par laser, mais posent aussi un grand nombre de questions. La comparaison avec la théorie se fait bien sur les aspects globaux, spectres, taux de conversion, mais les aspects temporels auxquels nous avons accès maintenant de façon plus précise, et qui sont très intéressants par leurs liaisons avec les phénomènes de transport de l'énergie et les opacités, révèlent des difficultés d'interprétation. Ces difficultés, pour être levées, nécessitent une étude plus approfondie de la physique atomique hors équilibre thermodynamique et des opacités utilisées dans les codes, ainsi que la définition de nouvelles expériences.

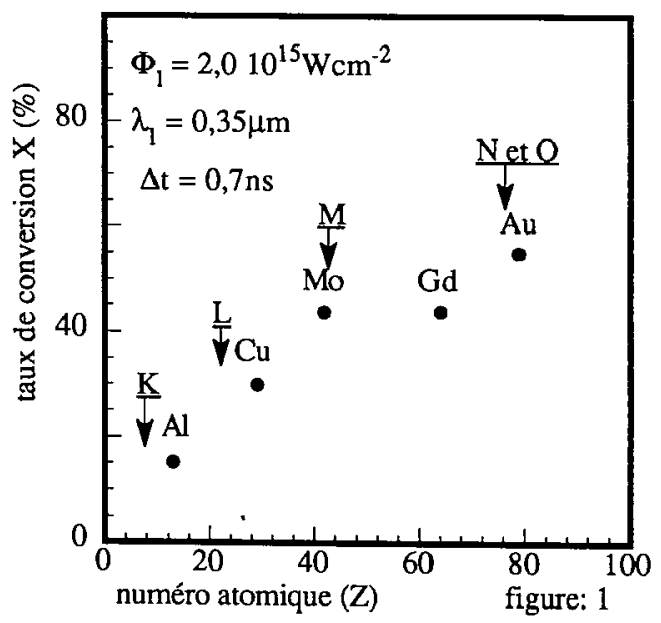




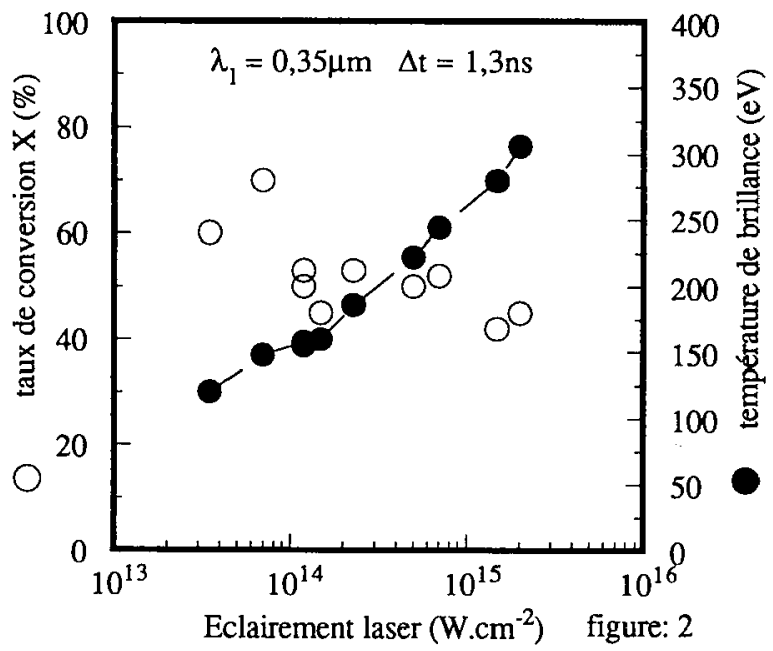

([1] D.BABONNEAU, J.L. BOCHER, CHOCS Revue Scientifique et Technique de la DAM N¹ (1991)) 